\title{
Teaching the electromagnetic spectrum with the Invisible Universe GEMS guide
}

\section{Stephen Pompea, Constance Walker, Erika Offerdahl}

Stephen M. Pompea, Constance E. Walker, Erika Offerdahl, "Teaching the electromagnetic spectrum with the Invisible Universe GEMS guide," Proc. SPIE 9663, Eighth International Topical Meeting on Education and Training in Optics and Photonics, 966325 (6 October 2003); doi: 10.1117/12.2208491

SPIE Event: Eighth International Topical Meeting on Education and Training in Optics and Photonics, 2003, Tucson, Arizona, United States 


\title{
Teaching the electromagnetic spectrum with the "Invisible Universe" GEMS guide
}

\author{
Stephen M. Pompea and Constance E. Walker \\ National Optical Astronomy Observatory, 950 N. Cherry Ave., Tucson, AZ 85719 \\ telephone: 520-318-8285; fax: 520-318-8451; e-mail: spompea@noao.edu \\ Erika Offerdahl \\ Steward Observatory, University of Arizona, Tucson, AZ 85721
}

\begin{abstract}
A new teacher's guide in the Great Exploration in Math and Science series, titled "Invisible Universe" addresses teaching approaches for the subject area of the electromagnetic spectrum and the sources and detectors needed for different wavelengths. This guide contains inquiry-oriented activities about waves, different parts of the electromagnetic spectrum, and the astronomical discoveries that can be made at each wavelength. As such it represents an integrated approach for this important optics subject matter area. Although the guide is written for teachers teaching grades 6-8 the activities can be used for high school and even college students.

(C) 2003 Optical Society of America

OCIS codes: (000.2060) Education; (350.1260) Astronomical Optics; (350-1270) Astronomy and Astrophysics
\end{abstract}

\section{Summary}

The GEMS book "Invisible Universe" explores a number of ways that the electromagnetic spectrum can be taught and includes well-tested inquiry oriented activities. This guide can serve as a model for teaching a variety of optical phenomena.

Like a horse with blinders, humans can see only a thin, restricted portion of the electromagnetic spectrum. One of the biggest challenges in teaching about the electromagnetic spectrum is increasing student awareness and experience with the "invisible universe". An effective vehicle for doing this is the teacher's guide, titled "Invisible Universe" co-authored by Pompea, in the Great Exploration in Math and Science (GEMS) series from the University of California Berkeley's Lawrence Hall of Science.

This Grades 6-8 teacher's guide is devoted to increasing awareness and unraveling misconceptions about the electromagnetic spectrum. It aligns itself with a wide variety of skills, concepts, themes, and mathematical strands that are closely related to the national science education standards. It addresses as well the nature of science and mathematics and the process by which research in these areas is done. Each classroom session in the guide is outlined with an overview, materials list, and preparation requirements, followed by clear, step-by-step directions. The guide also contains background information for the teacher, along with photographs and illustrations suitable for large group presentations. The guide is filled with comments on presentation strategies and practical advice to help the teacher, including advice from teachers who tested the units.

The guide is aligns with the concept of the learning cycle, which gives consideration to the stages in the learning process. National guidelines for the improvement of science education from the American Association for the Advancement of Science (AAAS) and the National Academy of Sciences (NAS) are based on a similar educational approach. Pedagogical issues associated with optics teaching in an exploratory manner will also be discussed.

The National Optical Astronomy Observatory is funded by the National Science Foundation under a cooperative agreement. The Invisible Universe guide was developed with the support of the NASA SWIFT Education and Public Outreach Program. More information on the GEMS program is available at http://www.lhsgems.org/gems.html 